\title{
Analysis of Influencing Factors on Air-Stable Organic Field-Effect Transistors (OFETs)
}

\author{
Yong PAN ${ }^{1}$, Aifeng LV ${ }^{1}$, Lifeng $\mathrm{CHI}^{2 *}$ \\ ${ }^{1}$ Physical Institute and Center for Nanotechnology (CeNTech), University Münster, Wilhelm-Klemm-Str. 10, Münster \\ 48149, Germany \\ ${ }^{2}$ Jiangsu Key Laboratory for Carbon-Based Functional Materials \& Devices, Functional Nano and Soft Materials \\ Laboratory (FUNSOM), Soochol University, Renai Rd. 199, Suzhou 215123, China
}

crossref http://dx.doi.org/10.5755/j01.ms.24.2.18197

Received 18 May 2017; accepted 05 July 2017

\begin{abstract}
The primary emphasis in this paper is on the major developments in the field of air-stable organic field-effect transistors (OFETs) over the past 20 years. The studies about the factors influencing the stability of OFETs, including air, humidity, oxygen and temperature, are reviewed and analyzed. The possible mechanisms that result in the degradation of OFETs, such as the penetrating of $\mathrm{H}_{2} \mathrm{O}$ molecules, the doping effect of oxygen or the crystalline structure difference caused by temperature, are summarized. At same time, the reason why the field-effect mobility and the on/off current ratio of the transistor might change greatly with different ambient is concluded. The overall lives of OFET-based sensor in the detection of hazardous gases including nitrogen dioxide and ammonia are discussed, several breakthrough findings and technologies about how to solve the problem of instability of OFETs are also presented.

Keywords: OFETs, stability, sensor, air, humidity, oxygen, temperature.
\end{abstract}

\section{INTRODUCTION}

During the past many years, the performance of organic field-effect transistors (OFETs) has been continually improved and become more and more possible in industrial applications [1-3]. As a new developing sensing platform, OFET-based sensors, due to their high sensitivity, low production costs, room temperature detection, have also attracted intriguing attention owing to their advantages of plenty organic material resources, mechanical flexibility, and microarray compatibility, but the problem of instability of OFETs is still the focus of researchers, especially the interface property of dielectrics. The influencing of oxygen, air, moisture, is the most important factors to influence the OFETs. Although these mechanisms are not confirmed, some people have involved in developing a high performance OFETs for many years and have made great efforts to explain the mechanisms [4-6]. Today, the OFET devices are currently being driven by two methods, the optimization of device structures and the development of new organic semiconductors, while the major challenge in OFETs research is the development of practical OFET materials that ensure not only high performance but also high stability in the operational environment, so the mechanism study influencing the stability of OFETs is surely urgent.

\section{AIR-STABLE ORGANIC FIELD-EFFECT TRANSISTORS}

The environmental instability of OFETs has been attributed to chemical impurities, molecular structure, ionization potential, broken conjugation, water, oxygen,

\footnotetext{
* Corresponding author. Tel.: +49-251-8333651; fax: +49-251-8333602.

E-mail address: chilf@suda.edu.cn (L.F.Chi)
}

and light exposure. With the development of conductive polymers whose highly conductive characteristic can be used as electrodes in OFETs, a lot of all-polymers OFETs have been studied. More than ten years ago, Henning et al. had reported on the fabrication of all-polymer OFET comprising conductive poly(ethylenedioxythiophene) poly(styrene sulfonic acid) systems (PEDOT-PSS), as electrode material, poly(3-hexylthiophene)I(P3HT) as semiconductor, as well as polymer substrates and dielectrics. The OFET was measured after 30 days storage under ambient oxygen and humidity conditions, it could be found that both the maximum on-current and the offcurrent rose greatly, but the on/off ratio thereby decreasing from 97 to 81 , they thought the good performance of devices was probably related with the top-gate setup and the purity of the materials chosen [7]. On the other hand, as most of organic semiconductors exhibited only single carrier (electron or hole) operation mode due to intrinsic impurities level, so the ambipolar characteristic had shown its potential as alternatives for the research of OFETs which could be operated either p- or n-type in a single device, but if the measurement processes were carried out in air ambient, the deterioration of electrical characteristics might result in [8].

The stability of organic semiconducting polymers toward oxidative doping is related to their ionization potentials, i.e., the highest occupied molecular orbital (HOMO) levels. Amanda et al. explored a method for increasing the stability of polythiophenes toward oxidative doping by lowering the HOMO energy level through the addition of electronwithdrawing alkyl carboxylate substituents [9], the regioregular ester-functionalized polythiophenes were found to have charge mobilities to $0.07 \mathrm{~cm}^{2} \mathrm{~V}^{-1} \mathrm{~s}^{-1}$. Later, Tomo Sakanoue investigated the electrical characteristics of single-component ambipolar 
OFETs by controlling the device structure and the measurement conditions, he found the hole mobility was barely affected by the exposure while the electron mobility was significantly affected [10].

To get the high $\mu_{\mathrm{FET}}$ of thin-film-based OFETs, which is the most important parameter in using, Kazuo et al. had ever synthesized DPh-BTBT, 2,7diphenyl[1]benzothieno[3,2-b][1] enzothiophene [11], this high-quality homogeneous thin films were easily obtained by vacuum deposition on the bare $\mathrm{Si} / \mathrm{SiO}_{2}$ substrates of top-contact-type OFET. Treated with octyltrichlorosilane (OTS) and fabricated at $100{ }^{\circ} \mathrm{C}$, the devices with $\mu_{\mathrm{FET}}=2.0 \mathrm{~cm}^{2} \mathrm{~V}^{-1 \mathrm{~s}-1}$ and $I_{\mathrm{on}} / I_{\mathrm{off}} \sim 10^{8}$ were reproducibly obtained, and the mobility showed a slight decrease after 250 days storage. Considering from the chemical structure, it was obvious that when the number of benzene rings increased, the HOMO level became higher and the HOMO-LUMO (Lowest Unoccupied Molecular Orbital) gap became narrower. Such high-lying HOMO level made the molecule susceptible to air-oxidation in ambient conditions, and narrow HOMO-LUMO gap would cause readily photo-induced excitation that might lead subsequent chemical reactions $[12,13]$. Yutaka et al. also designed and synthesized carbonyl-bridged bithiazole as a new electronegative unit for air-stable n-type OFET, which contributed both to lowering the lowest unoccupied molecular orbital energy level and to stabilizing the anionic species, the electron mobilities and the on/off ratios were $0.06 \mathrm{~cm}^{2} \mathrm{~V}^{-1} \mathrm{~s}^{-1}$ and $10^{6}$ respectively [14]. Electrochemical measurements and $\mathrm{X}$-ray analysis of $\mathrm{C}$ $\mathrm{BTz}$ revealed that the combination of carbonyl-bridging and electron-accepting thiazole rings contributes to a low LUMO energy level for charge-carrier transport. After 1 year storage under ambient condition, the results indicated that physically adsorbed oxygen and/or water did degrade the device performance, while the C-BTz molecule did have an intrinsic air stability when it was used as an active material for n-channel OFETs.

P3HT has been widely studied for its wide commercial availability and high carrier mobility, but it is not easy to measure P3HT-based OFET in ambient conditions because of its rapid undesirable interactions with atmospheric oxygen and moisture. If the P3HT-based OFET was prepared with chloroform and hexamethyldisilazane (HMDS), it took only about $10 \mathrm{~min}$ in air for the off current to increase by one order of magnitude. While if it was prepared by 1,2,4- trichlorobenzene (TCB) and OTS, only a slight degradation was caused after $120 \mathrm{~min}$ exposure to air [15]. In all the factors concerning air stability of P3TH, crystallization was very crucial because a tight and ordered packing of P3HT molecules would provide effective resistance to atmospheric oxygen and water molecules penetrating the film and doping the P3HT at the semiconductor-insulator interface. In addition, many solvents may lead to different molecular packing and interface dipoles, which might also affect the HOMO and influence the stability of OFETs [16]. In 2010, Yanmin Sun et al. studied four different interfaces [17]: bare $\mathrm{SiO}_{2}$, $\mathrm{SiO}_{2}$ modified with OTS ( $\left.\mathrm{SiO}_{2}-\mathrm{OTS}\right), \mathrm{SiO}_{2}$ coated a thin PMMA layer ( $\left.\mathrm{SiO}_{2}-\mathrm{PMMA}\right)$, and $\mathrm{SiO}_{2}$ substrate coated with PMMA and subsequently treated with OTS $\left(\mathrm{SiO}_{2}-\right.$ PMMA-OTS), very different air stability was observed by modification of the interface, and it was believed that the improved stability was a consequence of $\mathrm{H}$-bonding interactions the acidic $\mathrm{OH}$ groups on the silica surface and the basic carbonyl groups on PMMA.

As most of n-channel OFETs could not be operated well in air, but for application in the future, n-type organic semiconductors are highly desired. Yoshinobu et al. fabricated a n-channel OFET with a film of N,N'-bis(4-trifluoromethyl -benzyl) perylene-3,4,9,10tetracarboxylic diimide (PTCDI-TFB) which had two electron-withdrawing trifluoromethylbenzyl groups and was expected to have a high electron affinity [18]. By calculating, the LUMO level was to be $4.8 \mathrm{eV}$ and the electron affinity of PTCDI-TFB was also estimated to be $4.8 \mathrm{eV}$. Another approach to improve the stability of $\mathrm{n}$ channel OFETs was the use of organic compounds possessing strong electron-withdrawing units as the semiconducting materials, such as Fang-Chung Chen et al. improved the air stability of n-channel OTFTs based on N,N'-dioctyl-3,4,9,10-perylene tetracarboxylic diimide (PTCDI-C8) by modifying the device dielectric surface using hydroxyl-free polymer insulators [19], Fig. 1.
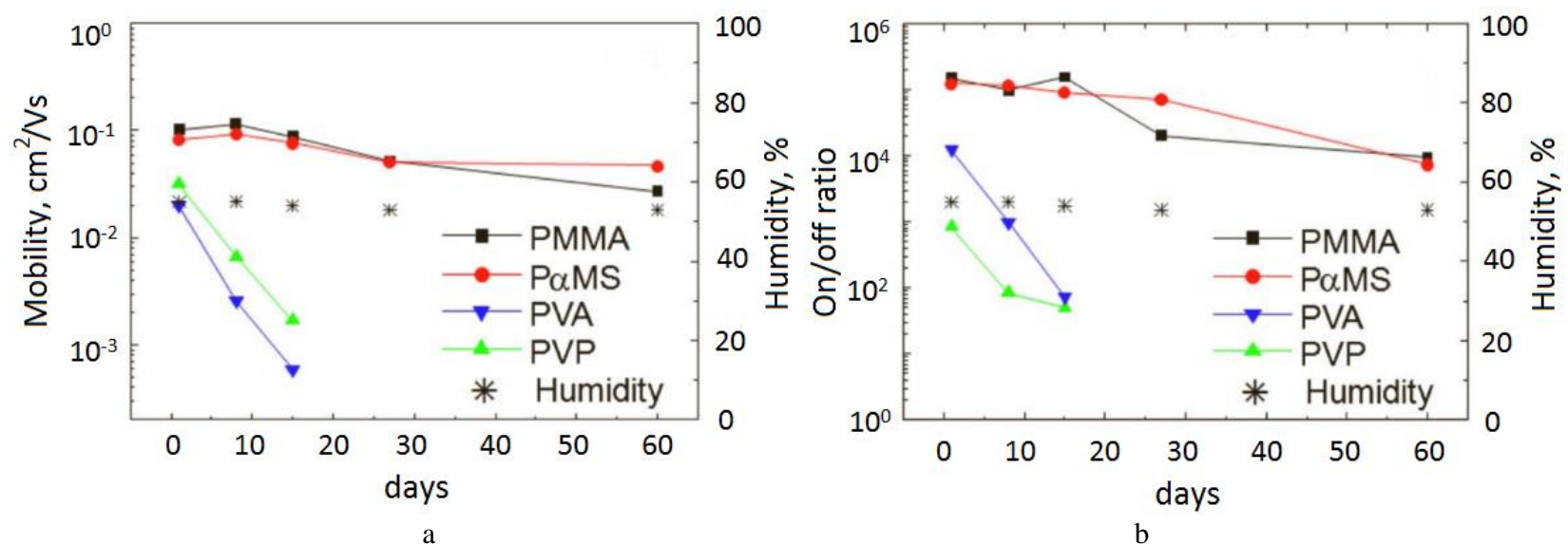

Fig. 1. Air-stability measurements of PTCDI-C8 OTFTs: a-electron mobilities and relative humidity as a function of time; b-on/off ratios plotted as a function of time. Between each measurement, the devices were stored in a cabinet, in which the relative humidity was also controlled at $50 \%-55 \%$ 
An example was that the poly(methyl methacrylate)(PMMA) and poly- $\alpha$-methylstyrene (P $\alpha \mathrm{MS}$ ) can improve the air stability of n-channel OTFTs because the complete absence of hydroxyl groups (electron traps) on the PaMS and PMMA surfaces led to enhanced nchannel conductionUnder ambient conditions, the electron mobility of the device incorporating PMMA as the modifying layer remained almost unchanged for the first 15 days and dropped to $0.03 \mathrm{~cm}^{2} \mathrm{~V}^{-1} \mathrm{~s}^{-1}$ after 60 days, he thought this method was general for n-channel OTFTs and could be used for other n-channel semiconductors.

To further illustrate the air stability of n-Channel OFET, more mechanisms were proposed and discussed. In general, thermodynamic stability of the charge carrier against ambient oxidants (e.g., $\mathrm{O}_{2}$ and $\mathrm{H}_{2} \mathrm{O}$ ) and the kinetic barrier provided by densely packed fluoroalkyl side chains $\left(-\mathrm{R}^{\mathrm{F}}\right)$ to keep $\mathrm{O}_{2}$ and moisture out of the active conducting layer are two much discussed rationales to account for the air stability of n-channel OFET, while a thermodynamically stable charge carrier (radical anion) is especially important for n-channel OFET. Experimentally, the first reduction potential $\left(E_{R 1}\right)$ of an n-channel organic semiconductor (OSC) molecule in solution was used to estimate its reduction propensity and to correlate with the ambient thermodynamic stability of a charge carrier (which actually exists in the solid state). Several threshold values of $E_{R 1}$ and $E_{\text {Lumo }}$ deduced from electrochemical measurements for air stability have been reported [20-22].

The charge carrier should be stable enough against ambient oxidants such as $\mathrm{O}_{2}$ and $\mathrm{H}_{2} \mathrm{O}$, and it has been suggested that OSCs with large enough electron affinity $\left(\mathrm{E}_{\mathrm{A}}\right)$ will possess air-stable charge carriers. Yu-Chang Chang et al. studied 47 existing n-channel OSCs with different molecular core structures and device configurations [23]. By analyzing datum, a correlation between calculated adiabatic $\mathrm{E}_{\mathrm{A}}$ and air stability was established at the level of theory, the minimum value of $E_{A}$ to achieve air stability against ambient oxidants is about $2.8 \mathrm{eV}$. For example, the $\mathrm{E}_{\mathrm{A}}$ of perylenetetracarboxylic diimides (PDI) was found to be close to the threshold value, therefore, it was not surprising that many PDI derivatives were air-stable. $\mathrm{E}_{\mathrm{A}}$ of the eight compounds demonstrating long-term stability were in the range of $2.8-3.7 \mathrm{eV}$ at the above theory level. Although the electron mobility might decrease to about $60 \%$ of its original value, some n-type OFET which could be easily solution-processed are fabricated with conventional techniques, and the on/off ratios remained almost unchanged after 30 days storage $[24,25]$. Heterotriangulene polymer (PTA) had ever been selected as an alternative organic semiconductor for air stable OFET, as the crucial air stability of PTA was provided by the low lying HOMO level and wide bandgap, which exhibiting an enhanced hole transporting ability in organic light emitting diodes (OLEDs) together with an improved thermal and morphological stability [26, 27]. Kerstin Schmoltner et al. reported an air-stable p-type PTA for large-area OFET applications, the synthesized amorphous organic semiconductor was characterized concerning morphological, optical, electrical and interface related properties and revealed a saturation mobility of about $4.2 \times 10^{-3} \mathrm{~cm}^{2} \mathrm{~V}^{-1} \mathrm{~s}^{-1}$ and an on/off current ratio of $\sim 10^{5}$ in bottom-gate/bottom-contact (BG/BC) OFETs. After storaging over 3 months, PTA OFETs did not show any significant changes in device performance, and the excellent stability was proved [28].

High HOMO energy level always leads to poor air stability, therefore, development of OFET materials with a low HOMO level and highly crystalline structure are need to overcome problem. In comparison with linear oligothiophenes, dendritic and hyperbranched oligothiophenes exhibited the relatively low-lying HOMO energy levels and could be used to improve air stability [29]. The best mobility of branched oligothiophenes was $0.012 \mathrm{~cm}^{2} \mathrm{~V}^{-1} \mathrm{~s}^{-1}$, it might provide extended delocalized $\pi$ electrons for enhancing charge transport [30]. Considering about this, Hung-Chin $\mathrm{Wu}$ et al. synthesized air stable branched octithiophene oligomer(8T) for OFET application [31], the 8T based OFETs showed the field effect mobility up to $2.12 \times 10^{-2} \mathrm{~cm}^{2} \mathrm{~V}^{-1} \mathrm{~s}^{-1}$ and a high on/off ratio of $5.1 \times 10^{6}$, the relatively low-lying HOMO energy level $(-5.53 \mathrm{eV})$ of $8 \mathrm{~T}$ led to the outstanding air stability of the OFET device, it was attributed to the fact that the octadecyltrichlorosilane (ODTS) - treated surface possessed a hydrophobic long alkyl chain, preventing the influence of oxygen and moisture [32].

Rizwan Ahmed et al. once studied the photoinduced charge transfer between C60 semiconductor layer and parylene dielectric [33]. He used the bottom-gate and topgate based C60 OFETs to compare the effects induced by light, it indicated clearly that the nature of the interface between the active layer and the dielectric played a major role in the light-induced threshold voltage shift, and it also proved that the light-induced threshold voltage shift strongly depended on the wavelength and intensity of the incident light as well as on the transverse electric field at the interface.

\section{THE FACTORS INFLUENCING OFETS}

\subsection{Influence of humidity on OFET stability}

In fact, most of sensors are affected easily by humidity and some can even be used as humidity sensor for their sensitivity to moisture $[34,35]$. Humidity can affect the charge transport properties of OFET in many different ways. Such as that $\mathrm{H}_{2} \mathrm{O}$ molecules can easily diffuse into these crevices and interact with the trapped carriers by altering the electric field at the grain boundaries, or the $\mathrm{H}_{2} \mathrm{O}$ molecules might be incorporated into the films and change their morphology, all these factors will affect the hole mobility and translate into changes in the saturation current, then devices show decreased current output and mobility as the relative humidity $(\mathrm{RH})$ is increased.

Degradation of organic thin-film field-effect transistors (OTFTs) with pentacene as the active material was studied by Yong Qiu et al., it was found that the fieldeffect mobility of the device decreased by $30 \%$ and the on/off current ratio decreased to one fifth after the OTFTs had been stored in atmosphere for $500 \mathrm{~h}$. AFM and IR spectrum analysis revealed that the degradation was caused by $\mathrm{H}_{2} \mathrm{O}$ molecules adsorbed on the pentacene film [36]. Atmospheric water might have a greater effect on the susceptibility of the device operating in air than $\mathrm{O}_{2}$. To 
prove this, OFET based on P3HT was prepared by Satoshi et al. to determine the influence of moisture on device characteristics [37]. The device was operated and measured under different conditions, by comparing the experiment results, an increase in the off-state conduction and a deterioration in the saturation behavior of the output $I_{\mathrm{d}}$ could be observed. It might be the adsorption of water molecules on the surface possibly increased the charge carrier density because of their relatively large dipole momentum.

In 2004, Bäcklund et al. investigated the hygroscopic insulator field-effect transistor (HIFET) fabricated from solution processable polymers. In his device, the current modulation was improved in the presence of moisture, the static performance of the device characterized by current level and modulation at low voltages (less than $1 \mathrm{~V}$ ) was greatly enhanced by moisture [38]. Later, they further clarified the influence of moisture and the effect of different solvents on the performance of the device by using a PEDOT:PSS(poly(3,4-ethylenedioxythiophene) doped with poly(styrene sulfonic acid)) gate electrode in different RH atmospheres [39]. The I-V characteristics were quite similar in RH around $40 \%-60 \%$, but changed significantly in lower humidity levels (RH $20 \%$ ), it might be the reason that the amount of the charge moving in the device increased with increasing humidity and the ions moved faster at higher humidity levels.

The device geometry (bottom or top contact device) and channel length are the other factors to influence the OTFT sensitivity by moisture. Dawen Li et al. selected a broader class of p-channel organic semiconductor materials including oligofluorene derivatives (C12FTTF and C6TFT) as well as pentacene as active layers for OTFTs, and two types of structures, i.e., top and bottom contact, with relatively large (200 microns) or small (25 microns) gaps between the source and drain electrodes were studied [40]. They found that OTFTs with small channel lengths and bottom contact structure had the highest sensitivity to $\mathrm{RH}$ by investigating the current response of an OTFT to humidity with different p-channel organic semiconductors and various device structures. They thought the reason was that the degradation of OTFT saturation current in a humid environment was expected to

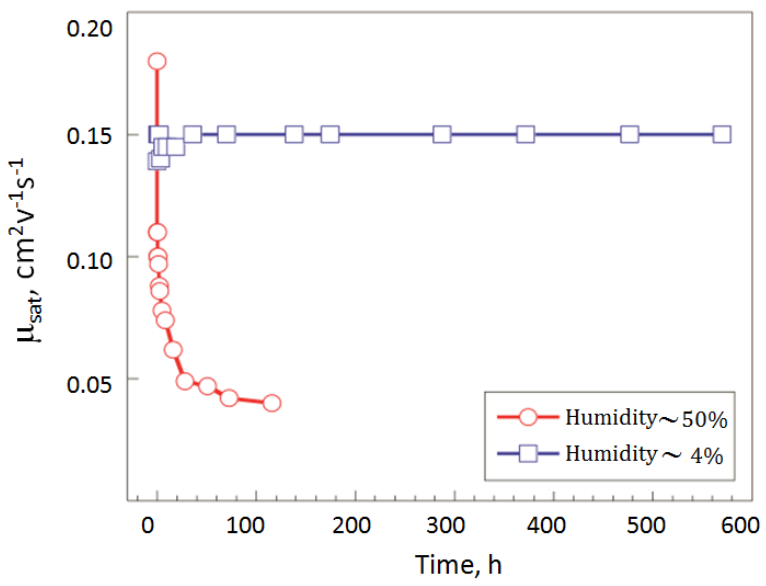

be strongly dependent on the density of grain boundaries between source and drain electrodes [41].

Organic semiconductors that can be fabricated by simple processing techniques and possess excellent electrical performance are very important in the preparing of OFET. The stability of the electrical performance of OFETs based on thieno[3,2-b]-thiophene polymer with an ionization potential of $5.10 \mathrm{eV}$ was discussed by McCulloch et al. [42]. After 5 days storage in ambient air with humidity $(\sim 50 \%)$, the field-effect mobility decreased by a factor of two, and the on/off ratio was reduced by two orders of magnitude. In comparison, the electrical performance of the device upon exposure to air with low humidity $(\sim 4 \%)$ showed the stability for more than 20 days, Fig. 2.

Fewer n-type materials used in OFET had been reported, moreover most of the reported n-type organic semiconducting materials showed instability and sensitivity to air exposure. Junhyuk Jang et al. selected CYTOPTM fluoropolymer as a dielectric insulator to reduce the effect of interface trap sites and fabricate ambient air-stable C60 OFETs [43]. His research indicated that the interface trap density for electrons for n-type organic materials was significantly reduced by using the perfluorinated polymer as the gate dielectric layer even in ambient air. To clarify the role of the water in combination with the polymeric semiconductor, electrical and optical techniques were used carefully by Nikolai Kaihovirta et al. to monitor the electrolyte/semiconductor interface in an ion conducting membrane based OFET. With the using of a hydrous ion conducting membrane (PTFE:PFSA), they found that the careful balancing of the hydration-level in the membrane was necessary in order to prevent excess gate leakage currents and a deterioration of the transistor characteristics, that meant a dry membrane would not induce charges to the active channel [44]. The effects of moisture on the electrical characteristics of pentacene field-effect transistors (FETs) with the polyvinylpyrrolidone (PVPy) gate insulator was also reported by Jaehoon et al., it was found that the device performance rapidly degraded following exposure to ambient moisture [45].

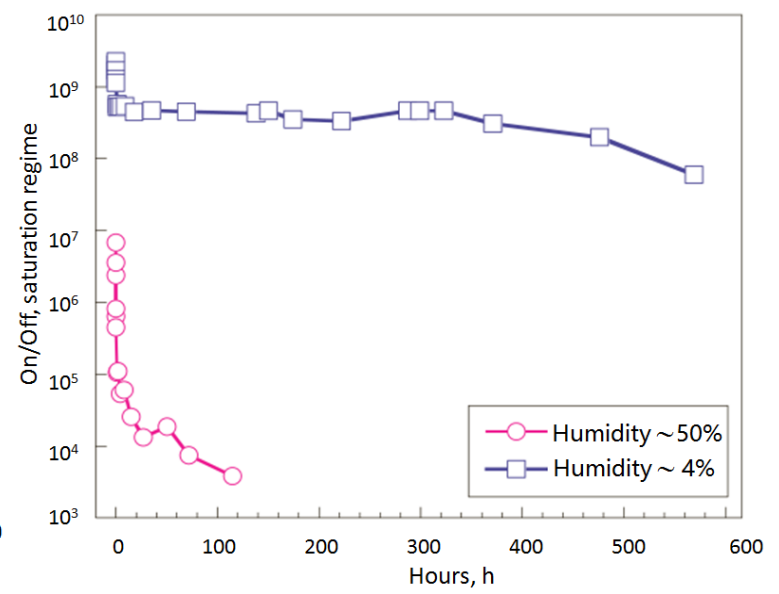

Fig. 2. Stability of FET devices. Transfer characteristics for polymer thieno[3,2-b]thiophene shown on prolonged exposure to change in mobility and ON/OFF ratio with time in both ambient and low-humidity air 
Se Hyun Kim et al. employed a $150 \mathrm{~nm} \mathrm{SiO}_{\mathrm{x}}$ film as an encapsulation layer to protect pentacene OFETs from $\mathrm{H}_{2} \mathrm{O}$, chemical and thermal damage [46].It could be found that the exposure of the $\mathrm{SiO}_{\mathrm{x}}$-capped device to ambient air could cause the decreases in $\mu_{\mathrm{FET}}$, the on-current and positive shift of the turn-on voltage, it implied that $\mathrm{H}_{2} \mathrm{O}$ molecules penetrating through the $\mathrm{SiOx}$ layer into the pentacene film and/or the pentacene/dielectric interface resulted in the degradation of device performances. Bovine serum albumin (BSA) was ever chosen as the gate dielectric material to fabricate OFETs and to illustrate its ability to increase the drain current at low voltage by Chun-Yi Lee et al. [47]. In a relative humidity of $47 \%$, the $\mu_{\text {FET,sat }}$ value increases from $0.3 \mathrm{~cm}^{2} \mathrm{~V}^{-1} \mathrm{~s}^{-1}$ to $4.7 \mathrm{~cm}^{2} \mathrm{~V}^{-1} \mathrm{~s}^{-1}$ and the $\mathrm{V}_{\mathrm{TH}}$ reduces from $-16 \mathrm{~V}$ to $-0.7 \mathrm{~V}$. The susceptibility to degradation in both ambient and aqueous environments could also prevented organic electronics from sensor using. To overcome this barrier, solution-processed OFETs which were based on a hygroscopic biomaterial with albumin as the gate dielectric and poly(3-hexylthiophene) as the semiconduct were fabricated by Mingchao Ma et al. [48]. It was proved that the device performance could be improved significantly with the increasing of $\mathrm{RH}$, and the results suggest moisture were potentially important in applications of biosensors.

\subsection{Influence of oxygen on OFET stability}

Molecular oxygen is a kind of strong electron acceptor leading to p-type conductivity of most organic semiconductors, so the charged p-type organic semiconductors are easily attacked in a reaction which can reduce water. Such as $\mathrm{P} 3 \mathrm{HT}$, a reversible decrease in the on/off-ratio of OFETs might be produced because of the enhanced bulk conductivity in the active P3HT layers [49]. In previous studies, the reversible and irreversible oxygen effects on polythiophenes (PT) was explained by the reversible formation of a charge-transfer (CT)-complex between molecular oxygen and the PT molecules [50], the existence of this metastable complex had been shown by its UV/Vis absorption band at the low energy edge of the P3HT absorption, but the information was not enough to completely understand the oxygen- induced effects in PT.

The methods to solve the problem of air stability of OFET were concluded as follow. First, kinetic barriers might limit the diffusion of air contamination to the channel region [51]. Second, thermodynamic control of the solubility of oxygen in the thin film was possible to tune the redox potentials of the organic semiconductors itself [52]. The third method was the device encapsulation by dielectric layers, which could also prevent the diffusion of oxygen and water vapors to the channel region of OFETs $[53,54]$. In 2004, L. LÜer et al. studied the oxygeninduced fluorescence quenching(FQ) of excited states of regioregular $\mathrm{P} 3 \mathrm{HT}$ in thin films to get some information about the mechanisms [55]. He thought the kinetics of FQ consisted of a fast component which was fully reversible, and a slow component which was partially irreversible, it was ascribed to collisional quenching of excited singlet states after oxygen diffusion into the bulk of the film and form reversibly charged states within $1 \mathrm{ps}$.

Toshio Nishi et al. investigated the interfacial electronic structure for titanyl phthalocyanine (TiOPc) prepared under ultrahigh vacuum (UHV) and $\mathrm{O}_{2}$ atmosphere by ultraviolet photoelectron spectroscopy (UPS) [56], the results indicated that the p-type semiconducting behavior of the TiOPc film deposited in $\mathrm{O}_{2}$, was consistent with the supposed p-type doping by $\mathrm{O}_{2}$ working as an electron acceptor. It was the first direct evidence of the doping effect by $\mathrm{O}_{2}$ based on the interfacial electronic structure with quantitative information about the relative position of Fermi level $\left(\mathrm{E}_{\mathrm{F}}\right)$ and the HOMO level, a clear conversion from n-type to p-type behavior was observed when the film was exposed to oxygen [57], X-ray photoelectron spectroscopy (XPS) of TiOPc film showed clearly the presence of extra oxygen forms for films deposited in moderate (10-6Torr) vacuums [58]. Two years later, the same working group of Toshio Nishi et al. studied the oxygen effect on the electronic structure of the interface between $\mathrm{C}_{60}$ and highly-oriented pyrolytic graphite by UPS, they noticed the removal of band bending near the interface, this change of the electronic structure and the deduced possible origins were consistent with the drastic decrease of the electrical conductivity of the $\mathrm{C}_{60}$ film fabricated in UHV at the exposure to $\mathrm{O}_{2}$, so the influence of the atmosphere on the charge transport and the device characteristic of $\mathrm{C}_{60}$ film was fatal [59].

The instability (oxidation sensitivity) of the compounds often lies in the low ionization potential, that is, a high-lying HOMO energy level. Within a particular class of compounds, an increased effective conjugation length leads to energy-rich HOMO levels and thus to an increased susceptibility towards oxidation [60]. Knipp et al. reported that the mobility of pentacene-based OFETs was not affected by exposure to dry $\mathrm{O}_{2}$ and decreased upon exposure to air with moisture. Yan et al. had also shown that some components of air including $\mathrm{N}_{2} / \mathrm{O}_{2}$ and $\mathrm{N}_{2} / \mathrm{H}_{2} \mathrm{O}$ had no effect on the electric characteristics of OFETs based on copper phthalocyanine $(\mathrm{CuPc})$ with a HOMO energy level of $-5.1 \mathrm{eV}$, these results indicated that wet oxygen was one of the factors causing the degradation of the performance of OFETs based on p-type organic semiconductors with high HOMO energy levels [61, 62].

The stability of OFETs based on two oligo-pphenylenevinylenes with different HOMO energy levels was studied by Tomoyuki. By experiments, the OFET with a HOMO energy level of $-5.55 \mathrm{eV}$ showed long-term air stability for a period exceeding one year, while the fieldeffect mobilities of organic semiconductors with a HOMO energy level ranging from -4.94 to $-5.20 \mathrm{eV}$ decreased with time, it meant that the organic semiconductors with a relatively low HOMO energy level had good chemical stability [63]. Daisuke Kumaki et al. paid attention on the influence of $\mathrm{O}_{2}$ and $\mathrm{H}_{2} \mathrm{O}$ on the transistor characteristics in the $\mathrm{p}$ - and n-type OTFTs fabricated on the $\mathrm{SiO}_{2}$ gate insulator without surface treatment of silane-coupling agent [64]. In both $\mathrm{p}$ - and n-type OTFTs, The $\mathrm{V}_{\text {th }}$ shifts to the positive direction indicated that negative charges were generated on the $\mathrm{SiO}_{2}$ gate-insulator surface by exposure to ambient air and dry air, the possibility that the electrochemical reaction at the interface was related to the deprotonation of $\mathrm{SiOH}$ with $\mathrm{O}_{2}$.

Bo-Chieh Huang et al. developed a simple technique 
(oxygen plasma treatment) to find the charge trapping measurement for OTFTs. They prepared the pentacenebased OTFTs with and without oxygen plasma treatment in order to display the relationship between charge trapping and transfer characteristics of OTFTs [65].In their works, the $\mu$ of OTFTs with oxygen plasma treatment was much higher than that of OTFTs without oxygen plasma treatment, and positive $\mathrm{V}_{\text {th }}$ shift was observed. In n-type OFETs, anions could form and had high reduction powers, so it could reduce oxygen molecules diffused into the layer of organic molecules. [66].

\subsection{Influence of temperature on OFET stability}

The influence of temperature is another important factor to the stability of OFETs. In 2000, Gilles Horowitz et al. performed current-voltage measurement on polycrystalline sexithiophene (6T) thin film transistors at temperatures ranging from 10 to $300 \mathrm{~K}$ [67]. They found that carrier mobility would increase quasilinearly with gate voltage at room temperature. Different substrate temperatures during the deposition process could influence the ordering and orientation of the molecules. While K.Xiao et al. observed high order and strong orientation of CuPc molecules on $\mathrm{Si} / \mathrm{SiO}_{2}$ by using XRD and TEM analysis at different substrate temperatures for a gate voltage $\mathrm{V}_{\mathrm{G}}=-50 \mathrm{~V}$ during the deposition process, which affected directly the morphology of thin films [68]. The channel conductivity was clearly thermally activated with a thermal activation energy, it was because of the defects, disorder and domain boundaries in the polycrystalline film. When the substrate temperature for deposition of $\mathrm{CuPc}$ reached to $120{ }^{\circ} \mathrm{C}$, a mobility of $3.75 \times 10^{-3} \mathrm{~cm}^{2} \mathrm{~V}^{-1} \mathrm{~s}^{-1}$ could be gotten.

It has been recognized that ordering of the organic film structure is very important for achieving high field-effect mobilities. The dependence $\mu(\mathrm{T})$ in pentacene thin film OFETs varies from thermally activated to temperature independent with $\mu(300 \mathrm{~K})$ increasing from $0.3 \mathrm{~cm}^{2} \mathrm{~V}^{-1} \mathrm{~s}^{-1}$ to $1.2 \mathrm{~cm}^{2} \mathrm{~V}^{-1} \mathrm{~s}^{-1}$ [69]. Podzorov et al. used parylene as a material as the gate insulator and fabricated the FETs at the surface of single crystals of rubrene. It demonstrated the hole-type conductivity with the field-effect mobility up to $1.0 \mathrm{~cm}^{2} \mathrm{~V}^{-1} \mathrm{~s}^{-1}$ and the on/off ratio up to $10^{4}$ at room

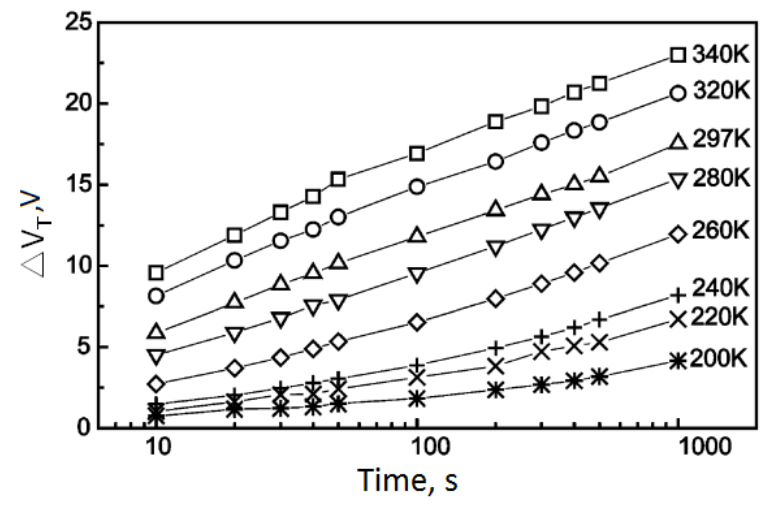

a temperature. The temperature dependence of the mobility depended strongly on the value of $\mu(300 \mathrm{~K})$ [70]. In 2004, Hamadani et al. reported his measurements of the parasitic contact resistance and the true channel resistance in bottom contact P3TH FET from room temperature down to $77 \mathrm{~K}$ [71], once parasitic contact resistances were taken into account, the mobility of solution-cast P3HT could approach $1.0 \mathrm{~cm}^{2} \mathrm{~V}^{-1} \mathrm{~s}^{-1}$ at room temperature, these results indicated that performance of P3HT-based OFETs could be limited more by contact physics than by the intrinsic tranport physics in the polymer itself. In 2004, Jianfeng Yuan et al. investigated the effects of OFET which used $\mathrm{F}_{16} \mathrm{CuPc}$ as active layer and tantalum pentoxide $\left(\mathrm{Ta}_{2} \mathrm{O}_{5}\right)$ as insulator[72], he demonstrated that the threshold voltage $\operatorname{shift}\left(\Delta \mathrm{V}_{\mathrm{T}}\right)$ was temperature dependent with activation energy of $0.51 \mathrm{eV}$, and the activation energy of $\Delta \mathrm{V}_{\mathrm{T}}$ might come from temperature dependent of hopping conduction in the insulator, Fig. 3.

The crystalline structure and the morphology of the organic semiconductor thin film can also be heavily influenced by the temperature (TD) at which the film is deposited. Th.B. Singh et al. investigated $\mathrm{C}_{60}$-based n-channel OFET with mobility in the range of $0.4-1 \mathrm{~cm}^{2} \mathrm{~V}^{-1} \mathrm{~s}^{-1}$. $\mathrm{C}_{60}$ OFETs exhibited a temperature dependent mobility with an Arrhenious behavior, and the devices characterised in inert atmosphere conditions showed high stability with an on/off ratio $>10^{4}$ [73]. Two years later, they extended an extensive study of morphology and crystallinity of the fullerene films using atomic force microscopy and grazing-incidence $\mathrm{x}$-ray diffraction, by experiments, they not only found the correlation of crystalline quality of the $\mathrm{C}_{60}$ film and charge carrier mobility, but proved that a higher substrate temperature led to single crystal-like faceted fullerene crystals [74]. To investigate the intrinsic transport mechanism of OFETs, Takeo Minari et al. developed a method of fabricating single-grain OFETs with top-contact structure by using finely formed metal mask. The results indicated the saturation mobility of $1.11 \mathrm{~cm}^{2} \mathrm{~V}^{-1} \mathrm{~s}^{-1}$ at $300 \mathrm{~K}$ increased with a decrease in temperature, reaching $1.22 \mathrm{~cm}^{2} \mathrm{~V}^{-1} \mathrm{~s}^{-1}$ at $210 \mathrm{~K}[75]$.

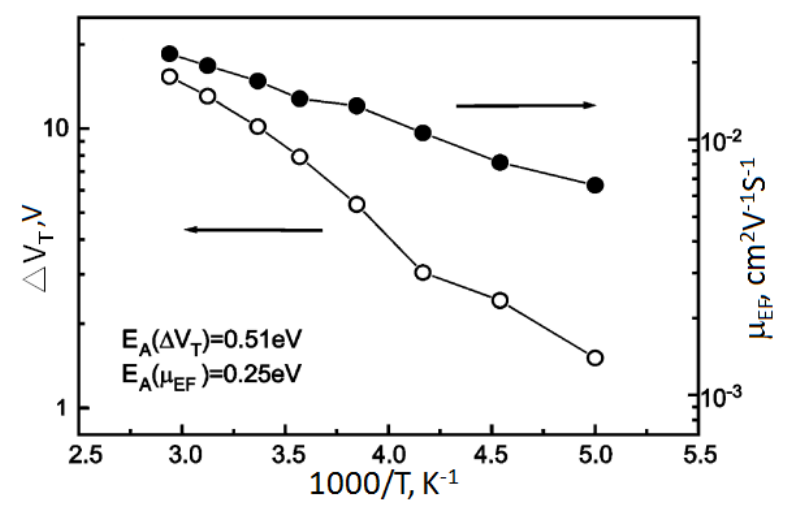

b

Fig. 3. $\mathrm{a}$ - time dependence of $\Delta \mathrm{V}_{\mathrm{T}}$ under various temperatures; $\mathrm{b}$-temperature dependence of the threshold voltage shift $\left(\mathrm{V}_{\mathrm{G}, \text { biass }}=50 \mathrm{~V}, \mathrm{t}=50 \mathrm{~s}\right)$ and the field-effect mobility 
In the aspect of mechanism, Lawrence Dunn et al. not only found that at higher temperatures $\mu_{\text {dynamic }}>\mu_{\mathrm{FE}}$, at lower temperatures $\mu_{\text {dynamic }}<\mu_{\mathrm{FE}}$, but also found $\mathrm{E}_{\mathrm{a} \text {,dynamic }}$ to be greater than $\mathrm{E}_{\mathrm{a}, \mathrm{FE}}$ by approximately $7 \mathrm{meV}$ at the same effective gate voltage [76].

It had been proven that the mobility of OFETs was determined by the layer-by-layer growth and the resultant crystallinity of the active layer, under a vacuum deposition condition, the structure of the organic semiconductor thin film was influenced by the growth conditions, e.g. substrate temperature, and so on. At a low temperature $\left(<90{ }^{\circ} \mathrm{C}\right)$, thin $(10-20 \mathrm{~nm})$ layers could easily obtained by hot-wire chemical vapour deposited (HWCVD) or solution-processed technologies [77, 78]. By this technology, high-performance OFETs with high-k dielectric for low-voltage, high field-effect mobility $(\mu)$ and on/off ratio could be fabricated. During the procedure, the film of OFET deposited at different temperature could be analysised by ultraviolet-visible-near-infrared (UVNIR) or X-ray diffraction patterns (XRD) [79]. In recently years, some people are focusing on biomaterial-based OFETs which can be used as temperature sensors because of the advantages including transparency, flexibility, thermal stability, high-temperature sensitivity and biocompatibility, and thus possess broad applicability for environmentally friendly electronics, implantable medical devices, etc [80].

\section{STABILITY OF THE OFETS SENSORS}

Many past and recent works successfully demonstrated the use of OFET in the field of gas sensing based on conjugated polymers, oligomers, or small molecules, such as the sensors used in humidity, ammonia, $\mathrm{NO}_{\mathrm{x}}$, formaldehyde, alcohol, explosive gas [81-84]. It is because, first, analyte molecules can diffuse through the semiconductor and approach the interface between the source and drain contacts, so the organic semiconductor can affect the carrier injection [85]. Secondly, the migration of analyte molecules into the organic semiconductor grain boundaries can deteriorate the carrier transport along the conduction channel of an OFET due to the introduction of localised charge traps [86], these interactions may have an impact on the conductance of the channel and the mobility of the carriers. Additionally, the analyte molecules that diffuse deeper into the semiconductor film and migrate at the semiconductordielectric interface of an OFET can also affect the threshold voltage of the device, so the stability of OFET sensors is still a serious problem in using.

Although many kinds of OFET sensors have been fabricated to detect different gases, the overall lifetime of the device for environmental stability is seldom reported. Junsheng $\mathrm{Yu}$ et al. created a kind of ammonia sensors based on bottom contact OFETs using pentacene as an active layer and polymerthylmethacrylate (PMMA) as an insulator. The variation of drain-source current was tested after 10 and 30 days storage in air when OFET sensor was exposed to different $\mathrm{NH}_{3}$ concentrations [87], Fig. 4.

In 2013, Liqiang Li et al. fabricated another ammonia gas sensor with ultrathin micro stripes of dialkyl tetrathiapentacene (DTBDT-C6) [81]. After storage in air for 25-35 days, the sensors still worked well and the sensitivity was still in the range of initial detection. These series of stability tests indicated the good reversibility and stability under continuous operation and storage conditions, which were rarely met in the OSCs-based sensors reported so far. The degrading of OFET sensor also happened on the detecting of dimethyl methylphosphonate (DMMP), after three months storing, although the trends of detection results to DMMP were consistent with the fresh preparing OFET sensors, the mobility value and the sensitivity would drop, and the response and recovery were rather slow [88].

In spite of the problems mentioned above, a lot of efforts are being made to optimized these OFET sensors. Minseong Yun et al. reported on a new sensor based on top-gate OFETs wherein chemical sensing was driven by the diffusion of ionic species. They demonstrated that topgate OFETs based on 6,13-bis(triisopropylsilylethynyl) pentacene (TIPS-pentacene) using a bilayer CYTOP/Al $\mathrm{O}_{2} \mathrm{O}_{3}$ dielectric could display excellent operational and environmental stability and were suitable for the implementation of reusable chemical/biological sensors because they primarily responded to charged species diluted in an aqueous media by rapidly shifting their threshold voltage [89].

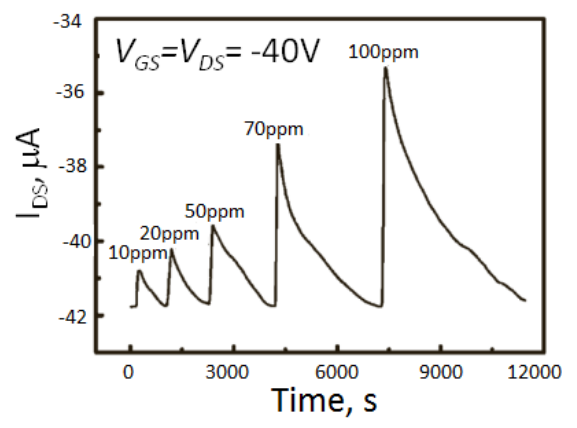

a

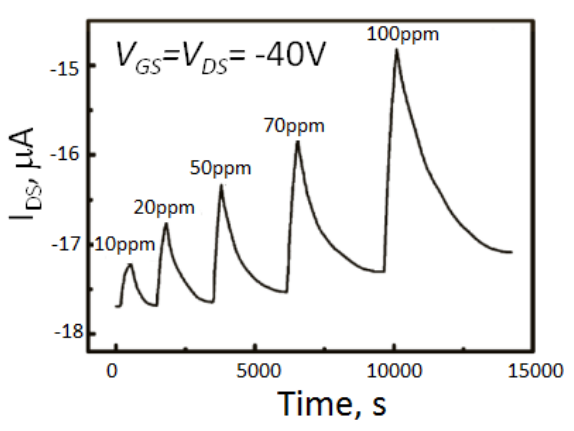

b

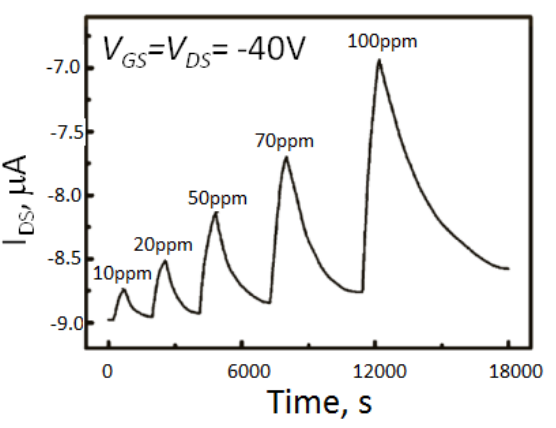

c

Fig. 4. Relationship between drain-source current and time when the OFET sensor exposed to various cycles of the exposure/evacuation different $\mathrm{NH}_{3}$ concentrations ranging from 10 to 100 ppm, the OFET sensor: $a$-detected $\mathrm{NH}_{3}$ after preparing; $b-$ was stored in air for 10 days; $c$ - was stored in air for 30 days 
Shijiao Han et al. fabricated a kind of high-response OFET-based $\mathrm{NO}_{2}$ sensor with the synergistic effect of zinc oxide/poly(methyl methacrylate) (ZnO/PMMA) hybrid dielectric and $\mathrm{CuPc} /$ Pentacene heterojunction, after being stored in atmosphere for 30 days, the variation of saturation current increased more than 10 times at $0.5 \mathrm{ppm}$ $\mathrm{NO}_{2}$, they thought the performance enhancement was due to the synergistic effect of the dielectric and organic semiconductor [90]. Although great achievement has been made, there are more and more experiments are needed to improve the stability of OFET sensors, the major findings of the studies in recent years have proved the fact that it is possible to solve the problems of stability of OFET sensor, of course, there is enormous research scope to extend the dimension of applicability in this field.

\section{CONCLUSIONS}

The present review attempts to give an overview of influencing factors of OFETs stored or operated in environment, and many research woks related with stability of OFET are summarized. From the discussion above, we find that almost all of the OFETs or OFETbased sensors are easily degraded by moisture, oxygen, temperature or other factors existed in ambient, such as the penetrating of $\mathrm{H}_{2} \mathrm{O}$ molecules, the doping effect of oxygen or the crystalline structure difference caused by temperature, field-effect mobility and the on/off current ratio of the transistor might decrease greatly because of the difference of ambient. Although there has been much development in designing and fabricating air-stable OFET to overcome this limitation during the past years, more studies are needed to understand device degradation mechanisms and improve the OFETs lifetimes. In conclusion, the stability of OFET is the most important in the application in the future, there is still enormous research scope to extend the dimension of applicability of OFETs.

\section{Acknowledgments}

This work was funded by the Germany-China Joint Project TRR61 (DFG-NSFC Transregio Project), the National Natural Science Foundation of China (91227201, 21527805) and Suzhou Industrial Park (SUN-WIN project 2013).

\section{REFERENCES}

1. Junsheng, Y., Xingge, Y., Lin, Z. Ammonia Gas Sensor Based on Pentacene Organic Field-Effect Transistor Sensors and Actuators B Chemical 173 2012: pp. 133-138. https://doi.org/10.1016/j.snb.2012.06.060

2. Henning, S. Device Physics of Solution-Processed Organic Field-Effect Transistors Advanced Materials 17 (20) 2005: pp. $2411-2425$. https://doi.org/10.1002/adma.200501152

3. Wei, H., Junsheng, Y., Xinge, Y. Polymer Dielectric Layer Functionality in Organic Field-Effect Transistor Based Ammonia Gas Sensor Organic Electronics 14 2013: pp. 3453-3459.

https://doi.org/10.1016/j.orgel.2013.09.018
4. Yaping, Z., Dazhen, H., Daoben, Z. Device Engineered Organic Transistors for Flexible Sensing Applications Advanced Materials 28 2016: pp. 4549-4555. https://doi.org/10.1002/adma.201505034

5. Anne-Marije, A., Christian, W.S., Michael, S. Localizing Trapped Charge Carriers in $\mathrm{NO}_{2}$ Sensors Based on Organic Field-Effect Transistors Applied Physics Letters 101 2012: pp. 153302. https://doi.org/10.1063/1.4758697

6. Misbah, M., Jiawei, W., Liang, W. Response Enhancement Mechanism of $\mathrm{NO}_{2}$ Gas Sensing in Ultrathin Pentacene Field-Effect Transistors Organic Electronics 24 2015: pp. $96-100$. https://doi.org/10.1016/j.orgel.2015.05.022

7. Rost, H., Ficker, J., Alonso, J.S. Air-Stable All-Polymer Field-Effect Transistors with Organic Electrodes Synthetic Metals 145 2004: pp. 83-85.

https://doi.org/10.1016/j.synthmet.2004.04.008

8. Jun, W., Haibo, W., Xuanjun, Y. Air-Stable Ambipolar Organic Field-Effect Transistors Based on Phthalocyanince Composites Heterojunction Chemical Physics Letters 407 2005: pp. $87-90$. https://doi.org/10.1016/j.cplett.2005.03.072

9. Amanda, R.M., Jinsong, L., Christine, L. Synthesis, Characterization, and Field-Effect Transistor Performance of Carboxylate-Functionalized Polythiophenes with Increased Air Stability Chemistry of Materials 17 2005: pp. $4892-4899$. https://doi.org/10.1021/cm050911d

10. Tomo, S., Masayuki, Y., Chihaya, A. Electrical Characteristics of Single-Component Ambipolar Organic Field-Effect Transistors and Effects of Air Exposure on Them Journal of Applied Physics 103 2008: pp. 094509. https://doi.org/10.1063/1.2919738

11. Kazuo, T., Hideaki, E., Katsuhiro, S. 2,7Diphenyl[1]Benzothieno[3,2-b]Benzothiophene, A New Organic Semiconductor for Air-Stable Organic Field-Effect Transistors with Mobilities up to $2.0 \mathrm{~cm}^{2} \mathrm{v}^{-1} \mathrm{~s}^{-1} \quad$ Journal of the American Chemical Society 128 2006: pp. $12604-12605$. https://doi.org/10.1021/ja0640521

12. Jean-Luc, B., David, B., Veaceslav, C. Charge-Transfer and Energy-Transfer Processes in $\pi$-Conjugated Oligomers and Polymers: A Molecular Picture Chemical Reviews 104 2004: pp. 4971-5004. https://doi.org/10.1021/cr040084k

13. Kazuo, T., Tatsuya, Y., Hideaki, E. Design Strategy for air-Stable Organic Semiconductors Applicable to HighPerformance Field-Effect Transistors Science and Technology of Advanced Material 8 2007: pp. 273-276.

14. Yutaka, I., Masashi, N., Makoto, K. Air-Stable n-Type Organic Field-Effect Transistors Based on Carbonyl-Bridged Bithiazole Derivatives Advanced Functional Materials 20 2010: pp. $907-913$. https://doi.org/10.1002/adfm.200901803

15. Majewski, L.A., Kingsley, J.W., Balocco, C. Influence of Processing Conditions on the Stability of Poly(3hexylthiophene)-Based Field-Effect Transistors Applied Physics Letters 88 2006: pp. 222108. https://doi.org/10.1063/1.2208938

16. Majewski, L.A., Song, A.M. Extended Storage Time of Poly(3-hexylthiophene) Field-Effect Transistors via Immersion in Common Solvents Journal of Applied Physics 102 2007: pp. 074515. https://doi.org/10.1063/1.2785011 
17. Yanmin, S., Xiaofeng, L., Shiwei, L. Polythiophene-Based Field-Effect Transistors with Enhanced Air Stability Organic Electronics 11 2010: pp. 351-355. https://doi.org/10.1016/j.orgel.2009.10.019

18. Yoshinobu, H., Daisuke, T., Hisao, I. Air-Stable n-Channel Organic Field-Effect Transistors Based on N,N'-bis(4Trifluoromethylbenzyl)perylene-3,4,9,10-tetracarboxylic diimide Chemical Physics Letters 436 2007: pp. $139-143$. https://doi.org/10.1016/j.cplett.2006.12.106

19. Fang-Chung, C., Cheng-Hsiang, L. Improved Air Stability of n-Channel Organic Thin-Film Transistors with Surface Modification on Gate Dielectrics Applied Physics Letters 93 2008: pp. 103310. https://doi.org/10.1063/1.2980421

20. Hakan, U., Chad, R., Zhimming, W. Design, Synthesis, and Characterization of Ladder-type Molecules and Polymers. Air-Stable, Solution-Processable n-Channel and Ambipolar Semiconductors for Thin-Film Transistors via Experiment and Theory Journal of the American Chemical Society 131 (15) 2009: pp. 5586-5608. https://doi.org/10.1021/ja809555c

21. Ming, L.T., Joon, H.O., Anna, D.R. Chlorination: A General Route Toward Electron Transport in Organic Semiconductors Journal of the American Chemical Society 131 (10) 2009: pp. 3733-3740. https://doi.org/10.1021/ja809045s

22. Rüdiger, S., Joon, H.O., Ya-Sen, S. High-Performance AirStable n-Channel Organic Thin Film Transistors Based on Halogenated Perylene Bisimide Semiconductors Journal of the American Chemical Society 131 (17) 2009: pp. $6215-6228$. https://doi.org/10.1021/ja901077a

23. Yu-Chang, C., Ming-Yu, K., Chih-Ping, C. On the Air Stability of n-Channel Organic Field-Effect Transistors: A Theoretical Study of Adiabatic Electron Affinities of Organic Semiconductors The Journal of Physical Chemistry C 114 (26) 2010: pp. 11595-11601. https://doi.org/10.1021/jp1025625

24. Luxi, T., Yunlong, G., Guanxin, Z. New Air-Stable Solution-Processed Organic n-Type Semiconductors Based on Sulfur-Rich Core-expanded Naphthalene Diimides Journal of Materials Chemistry 21 2011: pp. $18042-18048$. https://doi.org/10.1039/C1JM13637B

25. Yali, Q., Yunlong, G., $\quad$ Chunmeng, Y. Diketopyrrolopyrrole-Containing Quinoidal Small Molecules for High-Performance, Air-stable, and SolutionProcessable n-Channel Organic Field-Effect Transistors Journal of the American Chemical Society 134 2012: pp. $4084-4087$. https://doi.org/10.1021/ja3003183

26. Zhen, F., Vijila, C., Richard, D.W. Bridged-Triarylamine Starburst Oligomers As Hole Transporting Materials for Electroluminescent Devices Journal of Materials Chemistry 22 2012: pp. 15397-15404. https://doi.org/10.1039/C2JM32840B

27. Florian, S., $\quad$ Frédéric, R., $\quad$ Milan, K. $\pi$-Conjugated Heterotriangulene Macrocycles by Solution and SurfaceSupported Synthesis Toward Honeycomb Networks Journal of the American Chemical Society 135 2013: pp. $4550-4557$. https://doi.org/10.1021/ja400857g
28. Kerstin, S., Florian, S., Milan, K. A Heterotriangulene Polymer for Air-Stable Organic Field-Effect Transistors Polymer Chemistry 4 2013: pp. 5337-5344. https://doi.org/10.1039/C3PY00089C

29. Tsai, J.H., Lee, W.Y., Chen, W.C. New Two-Dimensional Thiophene-Acceptor Conjugated Copolymers for Field Effect Transistor and Photovoltaic Cell Applications Chemistry of Materials 22 (10) 2010: pp. 3290-3299. https://doi.org/10.1021/cm100661z

30. Achmad, Z., Askin, B., Frank, G. Solution Processable Organic Field-Effect Transistors Utilizing An $\alpha, \alpha$ Dihexylpentathiophene-based Swivel Cruciform Journal of the American Chemical Societ $128(12)$ 2006: pp. 3914-3915. https://doi.org/10.1021/ja0573357

31. Hung-Chin, W., Wen-Ya, L., Chih-Jung, L. Highly Air Stable Branched Octithiophene Oligomer for Organic Field Effect Transistor and $\mathrm{pH}$ Sensor Applications Materials Chemistry and Physics 138 2013: pp. 542-552. https://doi.org/10.1016/j.matchemphys.2012.12.017

32. Kumaki, D., Umeda, T., Tokito, S. Influence of $\mathrm{H}_{2} \mathrm{O}$ and $\mathrm{O}_{2}$ on Threshold Voltage Shift in Organic Thin-Film Transistors: Deprotonation of $\mathrm{SiOH}$ on $\mathrm{SiO}_{2}$ Gate-Insulator Surface Applied Physics Letters 92 2008: pp. 093309. https://doi.org/10.1063/1.2890853

33. Rizwan, A., Andrey, K., Clemens, S. Photosensitivity of Top Gate C60 Based OFETs: Potential Applications for High Efficiency Organic pPhotodetector Organic Electronics 15 2014: pp. 175-181. https://doi.org/10.1016/j.orgel.2013.11.002

34. Torsi, L., Dodabalapur, A., Cioffi, N., Sabbatini, L., Zambonin, P.G. NTCDA Orgnic Thin Film Transistor Humidity as Sensor Sensors and Actuators B 77 2001: pp. $7-11$. https://doi.org/10.1016/S0925-4005(01)00664-5

35. Zheng-Tao, Z. Jeffrey, T.M., Ru, D.D. Humidity Sensors Based on Pentacene Thin-Film Transistors Applied Physics Letters 81 (24) 2002: pp. $4643-4645$. https://doi.org/10.1063/1.1527233

36. Yong, Q., Yuanchuan, H., Guifang, D. $\mathrm{H}_{2} \mathrm{O}$ Effect on the Stability of Organic Thin-Film Field-Effect Transistors Applied Physics Letters 83 (8) 2003: pp. 1644-1646. https://doi.org/10.1063/1.1604193

37. Satoshi, H., Manabu, Y., Sei, U. Influence of Moisture on Device Characteristics of Polythiophene-Based Field-Effect Transistors Journal of Applied Physics 95 (9) 2004: pp. $5088-5093$.

https://doi.org/10.1063/1.1691190

38. Sandberg, H.G.O., Bäcklund, T.G., Österbacka, R. High Performance All Polymer Transistor Utilizing A Hygroscopic Insulator Advanced Materials (Weinheim, Ger.) 2004: pp. 1112-1115. https://doi.org/10.1002/adma.200400030

39. Bäcklund, T.G., Österbacka, R., Stubb, H. Operating Principle of Polymer Insulator Organic Thin-Film Transistors Exposed to Moisture Journal of Applied Physics 98, 2005: pp. 074504. https://doi.org/10.1063/1.2060949

40. Dawen, L., Evert-Jan, B., Robert, N. Humidity Effect on Electrical Performance of Organic Thin-Film Transistors Applied Physics Letters 86 2005: pp. $042105(1-3)$. https://doi.org/10.1063/1.1852708

41. Takao, S., Howard, E.K., Alan, G. Vapor Sensing with a,w-Dihexylquarterthiophene Field-Effect Transistors: The 
Role of Grain Boundaries Applied Physics Letters 81 (16) 2002: pp. 3079-3081.

https://doi.org/10.1063/1.1514826

42. Iain, M., Martin, H., Clare, B. Liquid-Crystalline Semiconducting Polymers with High Charge-Carrier Mobility Nature Materials 5 2006: pp. 328-333. https://doi.org/10.1038/nmat1612

43. Junhyuk, J., Kim, J.W., Nohhwal, P. Air Stable C60 Based n-Type Organic Field Effect Transistor Using A Perfluoropolymer Insulator Organic Electronics 9 2008: pp. $481-486$.

https://doi.org/10.1016/j.orgel.2008.02.011

44. Nikolai, K., Harri, A., Wikman, C.J. The Effects of Moisture in Low-Voltage Organic Field-Effect Transistors Gated with A Hydrous Solid Electrolyte Advanced Functional Materials 20 2010: pp. 2605-2610. https://doi.org/10.1002/adfm.201000586

45. Jaehoon, P., Doolee, S.B., Park, J. Effects of Moisture on Pentacene Field-Effect Transistors with Polyvinylpyrrolidone Gate Insulator Molecular Crystals and Liquid Crystals 531 2010: pp. 314-320. https://doi.org/10.1080/15421406.2010.495928

46. Se, H.K., Won, M.Y., Mi, J. Damage-free Hybrid Encapsulation of Organic Field-effect Transistors to Reduce Environmental Instability Journal of Materials Chemistry 22 2012: pp. $7731-7738$. https://doi.org/10.1039/C2JM13329F

47. Chun-Yi, L., Jenn-Chang, H., Yu-Lun, C. Hydrated Bovine Serum Albumin as the Gate Dielectric Material for Organic Field-Effect Transistors Organic Electronics 14 2013: pp. $2645-2651$. https://doi.org/10.1016/j.orgel.2013.07.004

48. Mingchao, M., Xinjun, X., Leilei, S. Organic Field-Effect Transistors with a Low Driving Voltage Using Albumin as the Dielectric Layer RSC Advances 4 2014: pp. 58720-58723. https://doi.org/10.1016/j.orgel.2013.07.004

49. Meijer, E.J. Dopant Density Determination in Disordered Organic Field-Effect Transistors Journal of Applied Physics 93 (8) 2003: pp. 4831-4835. https://doi.org/10.1063/1.1559933

50. Mohamed, S.A.A., $\quad$ Francesco, P.O., $\quad$ Yongkeun, S. Interaction of Oxygen with Conjugated Polymers:Charge Transfer Complex Formation with Poly(3-alkylthiophenes) Journal of the American Chemical Society 119 1997: pp. $4518-4524$. https://doi.org/10.1021/ja964229j

51. Howard, E.K., Jerainne, J., Andrew, J.L. Naphthalenetetracarboxylic Diimide-based n-Channel Transistor Semiconductors Journal of the American Chemical Society 122 (32) 2000: pp. 7787-7792. https://doi.org/10.1021/ja000870g

52. Thomas, D.A. Air-Stable Ambipolar Organic Transistors Applied Physics Letters 90 2007: pp. 122105.

53. Kim, S.H., Yoon, W.M., Jang, M. Damage-Free Hybrid Encapsulation of Organic Field-Effect Transistors to Reduce Environmental Instability Journal of Materials Chemistry 22 2012: pp. $7731-7738$. https://doi.org/10.1039/c2jm13329f

54. Seol, Y.G., Heo, W., Park. J.S. Improvement of Mechanical and Electrical Stability of Flexible Organic Field-Effect Transistors by Multistack Hybrid Encapsulation Journal of the Electrochemical Society 158(9) 2011: pp. H931 - H936. https://doi.org/10.1149/1.3610405
55. Lüer, L. Oxygen-Induced Quenching of Photoexcited States in Polythiophene Films Organic Electronics 5 2004: pp. $83-89$. https://doi.org/10.1016/j.orgel.2003.12.005

56. Toshio, N., Kaname, K., Yukio, O. Evidence for the Atmospheric p-Type Doping of Titanyl Phthalocyanine Thin Film by Oxygen Observed as the Change of Interfacial Electronic Structure Chemical Physics Letters 414 2005: pp. 479-482. https://doi.org/10.1016/j.cplett.2005.08.113

57. Hirokazu, T., Hiroshi, T., Masaki, T. Quasi-Intrinsic Semiconducting State of Titanyl-Phthalocyanine Films Obtained Under Ultrahigh Vacuum Conditions Applied Physics Letters 76 2000: pp. 873-875. https://doi.org/10.1063/1.125614

58. Klofta, T.J., Danziger, J., Lee, P. Photoelectrochemical and Spectroscopic Characterization of Thin Films of Titanyl Phthalocyanine: Ccomparisons with Vanadyl Phthalocyanine The Journal of Physical Chemistry 91 (22) 1987: pp. 5646-5651. https://doi.org/10.1021/j100306a030

59. Yusuke, T., Kaname, K., Yukio, O. Oxygen Effect on the Interfacial Electronic Structure of $\mathrm{C}_{60}$ Film Studied by Ultraviolet Photoelectron Spectroscopy Chemical Physics Letters 441 2007: pp. 63-67. https://doi.org/10.1016/j.cplett.2007.04.080

60. Sybille, A., Michael, F., Benjamin, S. Organic Semiconductors for Solution-processable Field-Effect Transistors (OFETs) Angewandte Chemie International Edition 47 2008: pp. 4070-4098. https://doi.org/10.1002/anie.200701920

61. Knipp, D., Benor, A., Wagner, V. Influence of Impurities and Structural Properties on the Device Stability of Pentacene Thin Film Transistors Journal of Applied Physics 2007: pp. 044504. https://doi.org/10.1063/1.2432369

62. Xuanjun, Y., He, W., Donghang, Y. An Investigation on Air Stability of Copper Phthalocyanine-Based Organic ThinFilm Transistors and Device Encapsulation Thin Solid Films 515 2006: pp. 2655-2658. https://doi.org/10.1016/j.tsf.2006.05.040

63. Tomoyuki, A., Takeshi, Y., Masatoshi, S. Air Stability of p-Channel Organic Field-Effect Transistors Based on Oligop-Phenylenevinylene Derivatives Japanese Journal of Applied Physics 47 (3) 2008: pp. 1760-1762. https://doi.org/10.1143/JJAP.47.1760

64. Daisuke, K., Tokiyoshi, U., Shizuo, T. Influence of $\mathrm{H}_{2} \mathrm{O}$ and $\mathrm{O}_{2}$ on Threshold Voltage Shift in Organic Thin-Film Transistors: Deprotonation of $\mathrm{SiOH}$ on $\mathrm{SiO}_{2}$ Gate-Insulator Surface Applied Physics Letters 92 2008: pp. 093309. https://doi.org/10.1063/1.2890853

65. Bo-Chieh, H., Yow-Jon, L. Effect of the Induced Electron Traps by Oxygen Plasma Treatment on Transfer Characteristics of Organic Thin Film Transistors Applied Physics Letters 99 2011: pp. 113301. https://doi.org/10.1063/1.3636411

66. Rizwan, A., Clemens, S. Air Stability of C60 Based n-Type OFETs Synthetic Metals 188 2014: pp. 136-139. https://doi.org/10.1016/j.synthmet.2013.12.007

67. Gilles, H., Mohsen, E.H., Riadh, H. Temperature and Gate Voltage Dependence of Hole Mobility in Polycrystalline Oligothiophene Thin Film Transistors Journal of Applied Physics 87 (9) 2000: pp. 4456-4463. https://doi.org/10.1063/1.373091 
68. Xiao, K., Liu, Y., Yu, G. Influence of the Substrate Temperature During Deposition on Film Characteristics of Copper Phthalocyanine and Field-Effect Transistor Properties Applied Physics A 77 2003: pp. 367-370. https://doi.org/10.1007/s00339-003-2169-6

69. Nelson, S.F., Lin, Y.Y., Gundlach, D.J. TemperatureIndependent Transport in High-mobility Pentacene Transistors Applied Physics Letters $72(15)$ 1998: pp. $1854-1856$. https://doi.org/10.1063/1.121205

70. Podzorov, V., Pudalov, V.M., Gershenson, M.E. FieldEffect Transistors on Rubrene Single Crystals with Parylene Gate Insulator Applied Physics Letters $82(11)$ 2003: pp. $1739-1741$. https://doi.org/10.1063/1.1560869

71. Hamadani, B.H., Natelson, D. Temperature-Dependent Contact Resistances in High-Quality Polymer Field-Effect Transistors Applied Physics Letters 84 (3) 2004: pp. $443-445$.

https://doi.org/10.1063/1.1639945

72. Jianfeng, Y., Jidong, Z., Jun, W. Study on the Instability of Organic Field-Effect Transistors Based on Fluorinated Copper Phthalocyanine Thin Solid Films 450 2004: pp. 316-319. https://doi.org/10.1016/j.tsf.2003.11.295

73. Singh, T.B., Marjanovic, N., Matt, G.J. High-Mobility nChannel Organic Field-Effect Transistors Based on Epitaxially Grown C60 Films Organic Electronics 6 2005: pp. $105-110$. https://doi.org/10.1016/j.orgel.2005.03.006

74. Singh, T.B., Sariciftc, N.S. Correlation of Crystalline and Structural Properties of C60 Thin Films Grown at Various Temperature with Charge Carrier Mobility Applied Physics Letters 90 2007: pp. 213512. https://doi.org/10.1063/1.2743386

75. Takeo, M., Takashi, N., Seiji, I. Temperature and ElectricField Dependence of the Mobility of A Single-Grain Pentacene Field-Effect Transistor Journal of Applied Physics 99 2006: pp. 034506. https://doi.org/10.1063/1.2169872

76. Lawrence, D., Ananth, D. Temperature Dependent Transient Velocity and Mobility Studies in An Organic Field Effect Transistor Journal of Applied Physics 107 2010: pp. 113714. https://doi.org/10.1063/1.3415546

77. Tiwari, S.P., Srinivas, P., Shriram, S. Organic FETs with HWCVD Silicon Nitride as a Passivation Layer and Gate Dielectric Thin Solid Films 516 2008: pp. 770-772. https://doi.org/10.1016/j.tsf.2007.06.048

78. Su, Y.R., Xi, W.G., Li, Y. A low-Temperature, SolutionProcessed High-k Dielectric for Low-Voltage, HighPerformance Organic Field-Effect Transistors (OFETs) Journal of Physics D: Applied Physics 46 2013: pp. 095105. https://doi.org/10.1088/0022-3727/46/9/095105

79. Liqiang, L., Qingxin, T., Hongxiang, L. An Ultra Closely p-Stacked Organic Semiconductor for High Performance
Field-Effect Transistors Advanced Materials 2007: pp. $2613-2617$.

https://doi.org/10.1002/adma.200700682

80. Xiaohan, W., Yan, M., Guoqian, Z. Thermally Stable, Biocompatible, and Flexible Organic Field-Effect Transistors and Their Application in Temperature Sensing Arrays for Artificial Skin Advanced Functional Materials 25, 2015: pp. $2138-2146$. https://doi.org/10.1002/adfm.201404535

81. Liqiang, L., Peng, G., Martin, B., Lifeng, C. Ammonia Gas Sensor Based on Pentacene Organic Field-Effect Transistor Advanced Materials 25 (25) 2013: pp. 3419-3425. https://doi.org/10.1002/adma.201301138

82. Necmettin, K., Ozturk, S., Atilla, D. Electrical and $\mathrm{NO}_{2}$ Gas Sensing Properties of Liquid Crystalline Phthallocyanine Thin Films Sensors and Actuators B 17 2012: pp. $203-210$. https://doi.org/10.1016/j.snb.2012.06.072

83. Bornali, B.P. Use of Organic Semiconductor in Gas Sensing International Journal of Advanced Research in Electrical, Electronics and Instrumentation Engineering $3(5)$ 2014: pp. 9351-9360.

84. Luisa, T., Maria, M., Kyriaki, M., Gerardo, P. Organic Field-Effect Transistor Sensors: A tutorial Review Chemical Society Reviews 42 2013: pp. 8612-8628. https://doi.org/10.1039/c3cs60127g

85. Roberts, M.E., Sokolov, A.N., Bao, Z.N. Material and Device Considerations for Organic Thin-Film Transistor Sensors Journal of Materials Chemistry $19(21)$ 2009: pp. $3351-3363$. https://doi.org/10.1039/b816386c

86. Torsi, L., Marinelli, F., Angione, M.D. Contact Effects in Organic Thin-Film Transistor Sensors Organic Electronics 10 (2) 2009: pp. 233-239. https://doi.org/10.1016/j.orgel.2008.11.009

87. Junsheng, Y., Xinge, Y., Lin, Z. Ammonia Gas Sensor Based on Pentacene Organic Field-Effect Transistor Sensors and Actuators B 173 2012: pp. 133-138. https://doi.org/10.1016/j.snb.2012.06.060

88. Kevin, C.S., Alan, B., Joseph, M. Enhanced Response of nChannel Naphthalenetetracarboxylic Diimide Transistors to Dimethyl Methylphosphonate Using Phenolic Receptors A Advanced Materials 19 2007: pp. 3322-3327. https://doi.org/10.1002/adma.200602924

89. Minseong, Y., Asha, S., Canek, F. Stable Organic FieldEffect Transistors for Continuous and Nondestructive Sensing of Chemical and Biologically Relevant Molecules in Aqueous Environment ACS Applied Material \& Interfaces 6 2014: pp. $1616-1622$. https://doi.org/10.1021/am404460j

90. Shijiao, H., Jiang, C., Huidong, F. Achievement of HighResponse Organic Field-Effect Transistor $\mathrm{NO}_{2}$ Sensor by Using the Synergistic Effect of ZnO/PMMA Hybrid Dielectric and $\mathrm{CuPc} /$ Pentacene Heterojunction Sensors 16 2016: pp. 1763(1-10). https://doi.org/10.3390/s16101763 